\title{
MUDANÇAS CLIMÁTICAS E A AGENDA DE ADAPTAÇÃO NAS CIDADES BRASILEIRAS 1
}

\section{CLIMATE CHANGE AND THE ADAPTATION AGENDA IN BRAZILIAN CITIES}

\author{
FABIANI BARBI \\ Pesquisadora de Pós Doutorado \\ Universidade Estadual de Campinas - UNICAMP \\ (Brasil) \\ fabarbi@gmail.com \\ Fernando Cardozo Fernandes ReI \\ Professor Associado do Programa de Doutorado em Direito Ambiental \\ Internacional \\ Universidade Católica de Santos (Unisantos) \\ (Brasil) \\ fernandorei@unisantos.br
}

Fecha de recepción: 24 de febrero de 2021 / Fecha de aceptación: 17 de mayo de 2021

RESUMO: As cidades e áreas urbanas são cada vez mais reconhecidas como arenas estratégicas para ações de mudança climática. Esse trabalho analisa como

1 Esse trabalho contou com o financiamento do Programa Nacional de Pós-Doutorado PNPD/Capes e apresenta resultados da pesquisa de pós-doutorado realizada no Programa de Pós-Graduação Stricto Sensu em Direito da Universidade Católica de Santos. 
as cidades brasileiras estão respondendo aos riscos das mudanças climáticas em termos de estratégias políticas de adaptação. Foi realizado um levantamento das cidades que possuem políticas climáticas aprovadas e dos planos de adaptação aos impactos das mudanças climáticas publicados no Brasil. A partir desses dados, foram extraídas e analisadas as principais variáveis que impulsionaram essas estratégias climáticas, com foco nos agentes e arranjos institucionais. Os resultados apontaram duas fases de ação climática para adaptação nas cidades brasileiras: na primeira, destaca-se a atuação das redes transnacionais de municípios, que trouxeram a questão climática para agenda política municipal e impulsionaram a capacidade de governança das mudanças climáticas dos governos locais brasileiros. Elas incitaram a formação dos comitês e fóruns municipais, que tiveram um papel fundamental na elaboração e aprovação das políticas climáticas. Na segunda fase, a ação adaptativa nas cidades tem se dado principalmente a partir do conhecimento acerca dos riscos climáticos nas localidades.

RESUM: Les ciutats i les zones urbanes són cada vegada més reconegudes com a escenaris estratègics per a accions de canvi climàtic. Aquest treball analitza com les ciutats brasileres responen als riscos del canvi climàtic en termes d'estratègies d'adaptació política. Es va dur a terme una enquesta de ciutats amb polítiques climàtiques aprovades $\mathrm{i}$ plans per adaptar-se als impactes del canvi climàtic publicada al Brasil. A partir d'aquestes dades, es van extreure i analitzar les principals variables que van impulsar aquestes estratègies climàtiques, centrant-se en els agents $\mathrm{i}$ en els arranjaments institucionals. Els resultats apunten a dues fases de l'acció climàtica per a l'adaptació a les ciutats brasileres: en la primera, destaca l'actuació de les xarxes transnacionals de municipis, que va portar el tema climàtic a l'agenda política municipal i va impulsar la capacitat de governança del canvi climàtic dels locals brasilers. governs. Van fomentar la formació de comitès $i$ fòrums municipals, que van jugar un paper fonamental en l'elaboració i aprovació de les polítiques climàtiques. En la segona fase, l'acció adaptativa a les ciutats es desenvolupa principalment a partir del coneixement sobre els riscos climàtics de les localitats. 
RESUMEN: Las ciudades y las áreas urbanas son cada vez más reconocidas como escenarios estratégicos para las acciones contra el cambio climático. Este trabajo analiza cómo las ciudades brasileñas están respondiendo a los riesgos del cambio climático en términos de estrategias de adaptación política. Se realizó una encuesta de ciudades con políticas climáticas aprobadas y planes de adaptación a los impactos del cambio climático publicados en Brasil. De estos datos se extrajeron y analizaron las principales variables que impulsaron estas estrategias climáticas, con especial atención a los agentes y arreglos institucionales. Los resultados apuntan a dos fases de la acción climática para la adaptación en las ciudades brasileñas: en la primera, se destaca el desempeño de las redes transnacionales de municipios, que llevaron el tema climático a la agenda política municipal e impulsaron la capacidad de gobernanza del cambio climático de los gobiernos. Impulsaron la formación de comités y foros municipales, que jugaron un papel fundamental en la elaboración y aprobación de las políticas climáticas. En la segunda fase, la acción adaptativa en las ciudades se da principalmente a partir del conocimiento sobre los riesgos climáticos en las localidades.

ABSTRACT: Cities and urban areas are increasingly recognized as strategic arenas for climate change actions. This work analyzes how Brazilian cities are responding to climate change risks in terms of adaptation policies. A survey was conducted with cities that have approved adaptation policies and plans to the impacts of climate change. Next, the main variables that drive these strategies were extracted and analyzed, focusing on agents and institutional arrangements. The results point to two phases of climate change adaptation actions in Brazilian cities: in the first, the highlights are transnational municipal networks, which have introduced the climate issue in the municipal political agenda and have fostered the climate governance capacity of local governments. These networks have also encouraged the formation of municipal climate committees and forums, which played a key role in the elaboration and approval of climate policies. In the second phase, adaptation actions in the cities take place mainly from the knowledge about climate-related risks in these localities. 
PALAVRAS-CHAVE: Mudanças climáticas - Políticas públicas - Riscos Cidades - Brasil.

PARAULES CLAU: Canvi climàtic - Polítiques públiques - Riscos - Ciutats Brasil.

PALABRAS ClAVE: Cambio climático - Políticas públicas - Riesgos Ciudades - Brasil.

KEYWORDS: Climate change - Public policies - Risks - Cities - Brazil.

SUMÁRIO: Introdução. 1. Riscos climáticos e a agenda de adaptação nas cidades. 2. A ação das Redes Transnacionais de Municípios nas cidades brasileiras. 3. Ações climáticas: políticas e planos de adaptação nas cidades brasileiras. 4. Agentes e arranjos institucionais para adaptação. Considerações finais. Referências.

\section{INTRODUÇÃO}

As mudanças climáticas se configuram como uma situação na contemporaneidade e é cada vez menos opcional responder aos riscos decorrentes dessas mudanças. Há evidências do aumento de eventos climáticos extremos em todo o mundo. Ademais, as projeções climáticas alertam que esses eventos deverão ser ainda mais frequentes e intensos, afetando a população com grandes perdas de vidas humanas, comprometendo a economia, a agricultura, a saúde e causando graves impactos nos ecossistemas (MAGRIN et al., 2014).

Qualquer adequação ou ajuste em resposta aos impactos reais ou previstos das mudanças climáticas, com os objetivos de lidar com as consequências, moderar os prejuízos, reduzir as vulnerabilidades ou explorar as oportunidades benéficas são caracterizados como estratégias de adaptação (ADGER et al., 2003; IPCC, 2007).

Em termos de respostas aos riscos climáticos, discute-se que os governos são agentes importantes que desempenham um papel fundamental na definição de normas, instituições e formas apropriadas de governança para enfrentar esses 
riscos em diferentes níveis e escalas (GIDDENS, 2009; BULKELEY, BETSILL, 2003; BULKELEY, NEWELL, 2010).

Nesse sentido, o nível local é considerado chave para responder aos desafios das mudanças climáticas. É crescente o reconhecimento do papel crucial das iniciativas locais no tratamento dos desafios climáticos, constituindo parte da dinâmica atual que sustenta o sistema climático e busca equilibrar as metas globais e as expectativas domésticas de ação (CONTIPELLI, 2018).

Nesse contexto, as cidades destacam-se entre os espaços mais vulneráveis aos impactos decorrentes das mudanças no sistema climático. Isso porque no século XXI, já chamado de "século urbano", mais da metade da população global é urbana e a expectativa é de rápido crescimento dessa população, especialmente nas cidades de países do sul global (HALL, PFEIFFER, 2013; HEYNEN, 2014; UN-HABITAT, 2016). Além disso, as cidades podem desempenhar um papel central no desenvolvimento e implementação de programas voltados ao enfrentamento das mudanças climáticas, pois estão localizadas na interface da ação local e dos compromissos de adaptação às mudanças climáticas em nível nacional e internacional (HEIDRICH et al., 2016).

Até o início do século $\mathrm{XXI}$, os debates na literatura e na prática se concentravam predominantemente na necessidade de mitigar as mudanças climáticas, isto é, de diminuir as concentrações de gases de efeito estufa (GEE) na atmosfera, que agravam as mudanças climáticas. Porém, a partir de então, a literatura e a prática têm colocado a necessidade de combinar os esforços de mitigação com a adaptação às mudanças climáticas, uma vez que os impactos dessas mudanças já estão sendo sentidos pelas populações e pelos ecossistemas em todo o mundo (ADGER et al., 2009; MEES, 2017). A literatura sobre governança adaptativa (MEES, 2017; AGRAWAL, 2008, MEASHAM, 2011) tem se concentrado na questão da agência, ou seja, no papel de diferentes atores públicos e privados na adaptação. Foi demonstrado que, na prática, geralmente são os governos locais que lideram a formulação de políticas locais concretas ou a tomada de ações específicas (HEGGER et al., 2017; MEES, 2017). 
Essa literatura tem abordado várias questões diferentes relacionadas à governança adaptativa conforme as regiões do mundo. No sul global, a literatura concentra-se na adaptação por e dentro das comunidades, isto é, adaptação baseada na comunidade (OLSSON et al., 2015). A literatura sobre o norte global concentra-se mais nas políticas de adaptação e nas ações de governos, famílias e cidadãos individuais. A liderança e participação dos governos locais, como nível institucional mais próximo dos cidadãos, desempenha um papel vital para qualquer compromisso sobre políticas de adaptação, por meio da sua incorporação bem-sucedida ao processo de planejamento do desenvolvimento urbano. É necessário entender as ações governamentais de adaptação aos impactos das mudanças climáticas no sul global, em termos de estratégias políticas, e essa é uma lacuna que esse trabalho pretende preencher, a partir do caso brasileiro.

No Brasil, mais de 160 milhões de pessoas vivem nas áreas urbanas, correspondendo a quase $85 \%$ da população (IBGE, 2010). O processo de urbanização nas cidades brasileiras trouxe desafios significativos para 0 planejamento urbano, como o esgotamento da infraestrutura, sobretudo dos sistemas de saneamento, transporte e habitação, além da deterioração ambiental (FERREIRA, 1996). Ainda, como consequência desse processo, há a ocupação de áreas consideradas inadequadas, expondo principalmente as camadas menos favorecidas da população a situações de risco como escorregamentos de terra e alagamentos (RIBEIRO, 2010). Esses problemas tendem a se intensificar com as mudanças climáticas, a partir do aumento do nível do mar, eventos extremos de calor e frio, períodos de estiagem e eventos extremos de precipitação, intensificando inundações e deslizamentos.

Assim, o objetivo principal desse trabalho é analisar como as cidades brasileiras estão respondendo aos riscos das mudanças climáticas em termos de estratégias políticas relacionadas à adaptação, contribuindo para o avanço do debate sobre governança climática no contexto brasileiro. 
Para tanto, em termos metodológicos, foi realizado um levantamento das políticas climáticas e dos planos de adaptação aos impactos das mudanças climáticas aprovados até 2020 nas cidades brasileiras. Em seguida, foram extraídas e analisadas as principais variáveis que contribuíram para essas estratégias climáticas. São elas: as redes transnacionais de municípios, os arranjos institucionais emergentes na governança climática urbana e os próprios riscos climáticos.

Primeiro, exploramos a agenda internacional de adaptação nas cidades. A seguir, discutimos o papel das redes transnacionais de municípios para a governança climática. Em seguida, analisamos as políticas e planos de adaptação nas cidades brasileiras, em suas particularidades e perspectivas. Por fim, analisamos os agentes e arranjos institucionais que surgem na governança climática nas cidades brasileiras.

\section{Riscos climáticos e a agenda de adaptação nas cidades}

A adaptação às mudanças climáticas passou a fazer parte da agenda pública no âmbito do regime internacional de mudanças climáticas já a partir da ConvençãoQuadro das Nações Unidas sobre Mudança do Clima (CQNUMC), em 1992, ainda que de forma subsidiária. Naquele momento, o foco da Convenção era a redução das emissões de GEE, o que determinou uma abordagem da problemática muito mais centrada em medidas de controle das emissões, ou seja, na sua mitigação. Contudo, os esforços não têm sido suficientes para alcançar a redução das emissões globais de GEE, que só diminuíram durante o ano de 2020 por conta da pandemia da Covid-19. De acordo com o IEA (2021), essas emissões devem aumentar em 2021 na recuperação econômica pós-Covid.

Assim, por algum tempo, não foi dada a atenção devida às políticas de adaptação aos impactos das mudanças climáticas, até mesmo porque, naquele momento, não eram tão frequentes e intensos os eventos extremos e havia certa percepção de que a redução das emissões seria capaz de lograr a estabilização das 
concentrações de GEE, num nível que impedisse uma interferência antrópica perigosa no sistema climático.

Além disso, para desenvolver estratégias de adaptação, uma base científica segura é fundamental (LINDSETH, 2005), já que implica em medidas com impacto nas searas ambiental, social e econômica dos territórios e devem ser pensadas e implantadas a partir da fundamentação no conhecimento científico, continuamente atualizado à luz de novas descobertas (REI, 2018). No entanto, mesmo com a expansão do conhecimento da ciência climática, as perdas por desastres relacionados ao clima têm aumentado globalmente, mostrando que os esforços de gestão de riscos naturais, reassentamento humano e redução de riscos de desastres não têm sido bem-sucedidas e ainda precisam de medidas políticas efetivas e investimentos (KLEIN et al., 2017).

Assim, diante dos impactos das mudanças climáticas, com o aumento da frequência e intensidade dos eventos climáticos extremos, a agenda global de adaptação e cidades ganhou reforços importantes na arena internacional. $O$ primeiro deles veio com os Objetivos do Desenvolvimento Sustentável (ODS), que fazem parte da agenda de desenvolvimento global aprovada pela ONU (Organização das Nações Unidas), em 2015, dos quais as cidades são um foco central.

Os dezessete ODS trazem diversas metas que estão alinhadas com o escopo da adaptação nas áreas urbanas, para o horizonte de 2030, como é o caso das metas relacionadas à água potável e saneamento (ODS 6), energia limpa e acessível (ODS 7), indústria, inovação e infraestrutura (ODS 9), redução das desigualdades (ODS 10), cidades e comunidades sustentáveis (ODS 11) e, em especial, ação contra a mudança global do clima (ODS 13).

Paralelamente, a Nova Agenda Urbana, resultante da conferência bi decenal do Programa das Nações Unidas para os Assentamentos Humanos (ONU-Habitat) 
em 2016, concentra-se fortemente no papel das cidades para a mitigação e adaptação às mudanças climáticas².

A adaptação climática nas cidades também possui estreita relação com a agenda de redução do risco de catástrofe e resiliência, difundida pelo Marco de Sendai (2015-2030) ${ }^{3}$ e pela Campanha Mundial 'Construir Cidades Resilientes' da ONU44, sobretudo no tocante à reverberação dessa agenda em estratégias municipais de Defesa Civil, que trazem a abordagem de gestão de riscos ligados ao clima.

Outro reforço na agenda de adaptação veio no âmbito do próprio regime internacional de mudanças climáticas, com o Acordo de Paris, também em 2015, resultante da Conferência das Partes (COP) 21, quando os Estados concordaram em fortalecer a capacidade das sociedades para lidar com os impactos das mudanças climáticas e fornecer apoio internacional contínuo para a adaptação nos países em desenvolvimento (UNFCCC, 2015). Nesta seara, inclusive o Acordo determina a revisão do trabalho de arranjos institucionais relacionados com a adaptação no âmbito da Convenção do Clima, com vista a identificar formas de aumentar a coerência do seu trabalho, a fim de responder adequadamente às necessidades dos Estados, bem como de seus entes descentralizados.

Dito de outra forma, as políticas de adaptação sugerem que o enfrentamento do problema na escala local requer a construção de uma lógica de governança multinível, numa dinâmica de complementaridade, permitindo que o avanço do regime também se dê pelas iniciativas desenvolvidas nos níveis intra e transnacionais e por atores ainda não formalmente partes do sistema jurídico internacional, os governos subnacionais de estados federados, bem como as cidades.

Afinal, líderes locais e regionais realizaram desde Johanesburgo, em 2002, cúpulas paralelas à COP, que acabaram, por força da atividade paradiplomática,

$2<$ http://habitat3.org/>, [última consulta em 10 de fevereiro de 2021].

3 https://www.undrr.org/implementing-sendai-framework/what-sendai-framework, [última consulta em 19 de abril de 2021].

${ }^{4}$ https://program.unisdr.org/campaign/resilientcities/, [última consulta em 19 de abril de 2021]. 
influenciando os informes e as próprias negociações internacionais sobre mudanças climáticas (HAPPAERTS et al., 2008; VAN DER HEIJDEN, 2018). E a participação de cidades na governança ambiental global ganha ainda mais força à medida que tais atores agem por meio de redes horizontais de cooperação (BULKELEY, BESTILL, 2003; SETZER, 2013).

Numa lógica completamente oposta ao Protocolo de Quioto, o Acordo de Paris marcou um novo modelo de contribuição dos países, não mais com imposição de metas, mas com contribuições voluntárias, na lógica bottom up, sendo que cada país indicou sua Contribuição Nacionalmente Determinada (NDC na sigla em inglês), com base nas suas capacidades domésticas (VICTOR, 2016). Ademais, essas contribuições indicaram não somente os planos dos países para reduzirem suas emissões de GEE, mas também descrições dos seus objetivos, prioridades, ações e necessidades de adaptação.

O objetivo do Acordo de "aumentar a capacidade adaptativa, fortalecer a resiliência e reduzir a vulnerabilidade às mudanças climáticas, com vistas a contribuir para o desenvolvimento sustentável e garantir uma resposta adequada de adaptação no contexto da meta de temperatura" vincula explicitamente a adaptação à meta de mitigação de limitar o aumento da temperatura global para bem abaixo de $2^{\circ} \mathrm{C}$. Essa conexão deixa claro que se as atividades de mitigação conseguirem, em tese, limitar o aumento da temperatura global, menos adaptação será necessária. Além disso, a cada cinco anos, os países revisarão e aumentarão a ambição de seus planos climáticos e enviarão e atualizarão periodicamente informações sobre suas prioridades de adaptação, implementação e necessidades de apoio a um registro público.

No que tange à adaptação, a NDC brasileira faz referência ao Plano Nacional de Adaptação à Mudança do Clima (PNA) como base das suas ações (BRASIL, 2015). O PNA foi publicado em 2016, sete anos depois da aprovação da Política Nacional de Mudança do Clima (PNMC), mostrando que no nível federal a questão da adaptação tarda a ser internalizada. Além disso, para os especialistas 
brasileiros (DI GIULIO et al., 2016), o PNA está aquém do necessário em relação à implementação, de fato, de políticas de adaptação.

Um dos pontos de destaque do Acordo de Paris foi o reconhecimento do papel das cidades, convidando-as a construírem resiliência e diminuírem a vulnerabilidade aos efeitos adversos das mudanças climáticas, além de defenderem e promoverem a cooperação regional e internacional. Esse aspecto está em consonância com grande parte da literatura acerca da governança climática (BULKELEY, BESTILL, 2003; HAPPAERTS et al., 2008; REl et al., 2012; SETZER, 2013), ao reconhecer a importância dos governos locais na implantação de estratégias políticas para tratar dos impactos das mudanças climáticas nas cidades. Eles concentram as principais responsabilidades de planejamento, implementação e gestão de uma grande parte das medidas que pode diminuir os riscos e as vulnerabilidades dos impactos diretos e indiretos das mudanças climáticas, através do fornecimento de infraestrutura e serviços, da preparação para desastres e da estrutura de planejamento e regulação (HUQ et al., 2007; SATTERTHWAITE, 2008).

Os primeiros experimentos de governança climática no mundo reconhecidos na literatura se deram através da ação municipal e das redes municipais transnacionais, como veremos na sequência.

\section{A ação das Redes Transnacionais de Municípios nas cidades brasileiras}

Há evidências suficientes na literatura (BETSILL, BULKELEY, 2004; TOLY, 2008; BOUTELIGIER, 2015; FÜNFGELD, 2015; HICKMANN, 2017; BELLINSON, 2018; RASHIDI, PATT, 2018) que as redes transnacionais de municípios (RTMs) possuem um papel importante na governança climática em todo o mundo, principalmente motivando os governos locais a definir e desenvolver iniciativas de políticas climáticas e ajudando-os na implementação dessas estratégias. As RTMs propiciam aos governos locais inspiração, informação, experiência, capacidades, projetos concretos, acesso a financiamento, exemplos de boas práticas e 
estruturas informais de reconhecimento e recompensas, que têm levado a respostas significativas para o desafio das mudanças climáticas.

As RTMs promoveram o desenvolvimento de uma abordagem explicitamente urbana à governação climática desde o início dos anos 90 (SCHROEDER, BULKELEY, 2009) e a trajetória das suas ações pode ser dividida em três fases. A primeira fase foi fundamental para elevar o perfil das cidades na governança ambiental, apesar de terem um impacto limitado na governança climática global. Foi orientada para o engajamento simbólico estimulado pela CQNUMC em $1992 \mathrm{e}$ pela formalização de esforços para desenvolver respostas conjuntas às mudanças climáticas (BULKELEY, 2010; GORDON, ACUTO, 2015). O ICLEI-Governos Locais pela Sustentabilidade, a Climate Alliance e a Energy Cities tiveram um papel estratégico e organizacional fundamental para a estruturação dessas redes. Esses primeiros esforços concentraram-se principalmente na eficiência energética nas operações municipais visando à mitigação das mudanças climáticas (HARVEY, 1993).

A segunda fase se deu desde o início dos anos 2000 (BULKELEY, BETSILL, 2013; BULKELEY et al., 2014; GORDON, ACUTO, 2015), quando essas redes se tornaram mais abrangentes e politicamente significativas na sua abordagem à governança do clima urbano. O destaque dessa fase é para a rede de cidades C40, criada em 2005, que reuniu as quarentas cidades mais populosas do mundo. A ênfase principal das redes nessa fase foi o desenvolvimento local e regional de infraestrutura e energia, uma preocupação grande dos países desenvolvidos, onde a maioria dessas redes atua (BULKELEY et al., 2014). As cidades continuaram seus esforços para se estabelecerem como atores climáticos capazes de ações eficazes, dentro da estrutura da CQNUMC, através de uma abordagem complementar à governança climática (HICKMANN, 2017).

A terceira fase, mais recentemente, caracteriza-se por uma maior atenção às estratégias de adaptação, impulsionadas principalmente pelas cidades do sul global. O surgimento da Asian Cities Climate Change Resilience Network (ACCRN), em 2008 e 100 Resilient Cities, em 2013, que passou a se chamar 
Resilient Cities Network (R-Cities), reforça o crescente interesse e necessidade de ações de adaptação e construção de resiliência em áreas urbanas. Alguns estudos sugerem que as RTMs estão se tornando importantes catalisadores para a adaptação local e podem acelerar e melhorar os processos de adaptação, assim como fizeram para a mitigação (FUNFGELD, 2015).

Nas últimas duas décadas, muitas cidades brasileiras também se envolveram em RTMs, particularmente naquelas que adotam políticas de sustentabilidade e mudanças climáticas. Pelo menos cinquenta governos subnacionais brasileiros são membros de seis das oito principais redes, e vários deles participam de mais de uma, como a cidade do Rio de Janeiro, que é membro de todas as redes (ver Tabela 1).

Tabela 1 - As principais redes transnacionais de municípios para as mudanças climáticas (em ordem cronológica) e a participação das cidades brasileiras

\begin{tabular}{|c|c|c|c|c|c|}
\hline Rede & $\begin{array}{l}\text { Início das } \\
\text { atividades } \\
\quad(a n o)\end{array}$ & $\begin{array}{l}\text { Número } \\
\text { de } \\
\text { cidades } \\
\text { membro }\end{array}$ & $\begin{array}{l}\text { Número } \\
\text { de países }\end{array}$ & $\begin{array}{l}\text { Número } \\
\text { de } \\
\text { cidades } \\
\text { brasileiras }\end{array}$ & $\begin{array}{c}\text { Principais } \\
\text { objetivos/programas }\end{array}$ \\
\hline $\begin{array}{l}\text { Metropolis } \\
\text { www.metropolis.org }\end{array}$ & 1985 & 139 & 64 & 7 & $\begin{array}{l}\text { Promover } \\
\text { desenvolvimento } \\
\text { urbano sustentável de } \\
\text { regiões metropolitanas. }\end{array}$ \\
\hline $\begin{array}{l}\text { Climate Alliance } \\
\text { www.climatealliance.org }\end{array}$ & 1990 & $1.871^{5}$ & 27 & 0 & $\begin{array}{l}\text { Projeto AMICA: } \\
\text { Integrar ações de } \\
\text { mitigação e adaptação } \\
\text { nas cidades; Aliança }\end{array}$ \\
\hline
\end{tabular}

${ }^{5}$ Incluindo: cidades, municípios e distritos, bem como províncias, ONGs e outras organizações de toda a Europa <https://www.climatealliance.org/municipalities/the-network.html>, [última consulta em 10 de fevereiro de 2021]. 


\begin{tabular}{|c|c|c|c|c|c|}
\hline Rede & $\begin{array}{l}\text { Início das } \\
\text { atividades } \\
\text { (ano) }\end{array}$ & $\begin{array}{l}\text { Número } \\
\text { de } \\
\text { cidades } \\
\text { membro }\end{array}$ & $\begin{array}{l}\text { Número } \\
\text { de países }\end{array}$ & $\begin{array}{l}\text { Número } \\
\text { de } \\
\text { cidades } \\
\text { brasileiras }\end{array}$ & $\begin{array}{c}\text { Principais } \\
\text { objetivos/programas }\end{array}$ \\
\hline & & & & & $\begin{array}{l}\text { Climática de Cidades } \\
\text { Europeias com Povos } \\
\text { Indígenas } \\
\text { Florestas. }\end{array}$ \\
\hline $\begin{array}{l}\text { Energy Cities } \\
\text { energycities.org }\end{array}$ & 1990 & 1.000 & 30 & 0 & $\begin{array}{l}\text { Pacto dos Prefeitos: } \\
\text { reduzir as emissões de } \\
\text { GEE em } 20 \% \text { até } 2020 . \\
2000 \text { cidades em } 42 \\
\text { países assinaram o } \\
\text { pacto até } 2010 . \\
30 \text { Propostas de } \\
\text { Transição Energética } \\
\text { nas Cidades. }\end{array}$ \\
\hline $\begin{array}{l}\text { ICLEI - Governos } \\
\text { Locais pela } \\
\text { Sustentabilidade } \\
\text { iclei.org }\end{array}$ & 1991 & 1.750 & $124^{6}$ & 50 & $\begin{array}{l}\text { CCP - Cidades pela } \\
\text { Proteção do Clima. }\end{array}$ \\
\hline $\begin{array}{l}\text { United Cities and } \\
\text { Local } \\
\text { Governments } \\
\text { (UCLG) } \\
\text { www.uclg.org }\end{array}$ & 2004 & 1.000 & 140 & 15 & $\begin{array}{l}\text { Parceria para Redução } \\
\text { de Risco Urbano; Pacto } \\
\text { dos Prefeitos para } \\
\text { desenvolver políticas } \\
\text { de proteção do clima } \\
\text { mundial. }\end{array}$ \\
\hline Climate & 2005 & 97 & 56 & 4 & $\begin{array}{l}\text { Adotar medidas para } \\
\text { redução de emissões }\end{array}$ \\
\hline
\end{tabular}

${ }^{6}$ Os membros do ICLEI em todo o mundo são principalmente cidades. No Brasil, alguns Estados também são membros da rede (Amazonas, Minas Gerais e São Paulo). 


\begin{tabular}{|c|c|c|c|c|c|}
\hline Rede & $\begin{array}{l}\text { Início das } \\
\text { atividades } \\
\text { (ano) }\end{array}$ & $\begin{array}{l}\text { Número } \\
\text { de } \\
\text { cidades } \\
\text { membro }\end{array}$ & $\begin{array}{l}\text { Número } \\
\text { de países }\end{array}$ & $\begin{array}{l}\text { Número } \\
\text { de } \\
\text { cidades } \\
\text { brasileiras }\end{array}$ & $\begin{array}{c}\text { Principais } \\
\text { objetivos/programas }\end{array}$ \\
\hline $\begin{array}{l}\text { Leadership Group } \\
\text { www.c40.org }\end{array}$ & & & & & $\begin{array}{l}\text { de GEE e mais } \\
\text { recentemente lidar com } \\
\text { riscos das mudanças } \\
\text { climáticas. }\end{array}$ \\
\hline $\begin{array}{l}\text { Asian Cities } \\
\text { Climate Change } \\
\text { Resilience } \\
\text { Network (ACCRN) } \\
\text { www.acccrn.net }\end{array}$ & 2008 & 40 & 6 & 0 & $\begin{array}{l}\text { Construir cidades } \\
\text { resilientes. }\end{array}$ \\
\hline $\begin{array}{l}\text { Resilient Cities } \\
\text { Network } \\
\text { resilientcitiesnetwork.org }\end{array}$ & 2013 & 100 & 47 & 3 & $\begin{array}{l}\text { Ajudar as cidades a se } \\
\text { tornarem } \\
\text { resilientes. }\end{array}$ \\
\hline
\end{tabular}

Fonte: BARBI, MACEDO, 2019.

Conforme a Tabela 1 mostra, a maior parte das cidades brasileiras faz parte do ICLEI. Essa rede posicionou as cidades brasileiras no debate sobre mudanças climáticas, trazendo a questão para suas agendas políticas locais em primeiro lugar. A agenda municipal de mudanças climáticas passa por avanços significativos desde o início de 2000 no Brasil, promovidos principalmente pela participação das cidades em projetos e alianças impulsionadas pelo ICLEI. Ao participarem de seus projetos e se juntarem a essa rede, cidades importantes do país tiveram mais informações sobre os desafios locais no enfrentamento da problemática, se engajaram em ações climáticas melhor elaboradas, prepararam e aprovaram políticas climáticas locais (BARBI, MACEDO, 2019).

O ICLEI adicionou questões relacionadas ao clima no escopo de ação dos municípios, como compras públicas sustentáveis, energias renováveis, construção sustentável, gerenciamento de resíduos sólidos e, mais recentemente, 
biodiversidade urbana. Isso tem se dado através do acesso a novos conhecimentos, criando um ambiente favorável ao desenvolvimento e aprovação de políticas climáticas locais e aumentando a eficácia percebida dessas políticas.

Os fatores de capacidade de governança climática dos governos locais brasileiros ganharam um reforço significativo com a participação deles na rede do ICLEI. Nos casos em que algum desses fatores (competência jurídica, recursos humanos e técnicos, financiamento) não exista em grau significativo, é provável que os governos locais não consigam implementar políticas de mudança climática (RYAN, 2015). No caso das cidades brasileiras, isso foi possível através das diversas funções desempenhadas pelo ICLEI ao longo de sua atuação, como: compartilhamento de informações, capacitação, construção de plataformas, consultoria, ação direta e implementação, definição de objetivos e regras e articulação.

Dessa forma, o ICLEI tem apoiado fortemente a ação climática nas cidades brasileiras sendo que a participação delas na rede teve um papel decisivo na adoção das suas políticas climáticas (BARBI, MACEDO, 2019). Até 2020, doze 7 dos 5.570 municípios brasileiros haviam aprovado suas políticas de mudança climática, sendo dez deles membros da rede. A seguir detalhamos essas políticas e planos de adaptação.

\section{Ações climáticas: políticas e planos de adaptação nas cidades brasileiras}

As ações climáticas nas cidades brasileiras dividem-se em duas fases principais. A primeira delas, até 2011 , é caracterizada pela reverberação da $15^{\text {a }}$ Conferência das Partes (COP) da CQNUMC ocorrida em 2009, em Copenhague. Naquele momento, a agenda política internacional voltava-se para a questão climática, com a publicação do Relatório de Avaliação do IPCC (2007) e com a mobilização da opinião pública internacional, influenciada pelo trabalho de Al Gore, principalmente

\footnotetext{
7 São eles: Belo Horizonte (MG), Curitiba (PR), Extrema (MG), Feira de Santana (BA), Fortaleza (CE), Manaus (AM), Palmas (TO), Porto Alegre (RS), Recife (PE), Rio de Janeiro (RJ), São Paulo (SP) e Sorocaba (SP).
} 
através do filme "Uma verdade inconveniente" (2006). Internamente, o governo federal brasileiro já vinha discutindo as bases para uma política climática desde 2000, através do Fórum Brasileiro de Mudanças Climáticas (FBMC). Assim, a Política Nacional de Mudanças Climáticas (lei no 12.187/09) foi aprovada em 2009, duas semanas após o término da 15 $5^{\mathrm{a}} \mathrm{COP}$. Com isso, o Brasil mudou sua posição histórica nas negociações internacionais e adotou uma meta voluntária para reduzir as emissões de GEE entre 38 e $36,1 \%$, ou seja, $9 \%$ do total de emissões projetadas para 2020 (BRASIL, 2009).

É nesse período que as primeiras políticas climáticas são aprovadas por cidades brasileiras: Palmas-TO (2003), Porto Alegre-RS (2007), Curitiba-PR (2009), São Paulo-SP (2009), Manaus-AM (2010), Belo Horizonte-MG (2011), Feira de Santana-BA (2011) e Rio de Janeiro-RJ (2011).

Entre 2011 e 2014 não ocorreu a aprovação de nenhuma política climática no Brasil no nível municipal. É um período em que o tema envolvendo as mudanças climáticas perde protagonismo na agenda política internacional, em decorrência dos efeitos da crise financeira internacional e pelas dificuldades de se avançar nas negociações de um novo período de compromissos, pós-Quioto, e isso se reflete nas cidades brasileiras também.

A segunda fase de ação climática nas cidades brasileiras, iniciada em 2014, é marcada por uma maior atenção ao aspecto da adaptação aos impactos das mudanças climáticas. Assim como na primeira fase, essa também recebe a influência do contexto internacional, quando o IPCC lança o seu $5^{\circ}$ Relatório de Avaliação (2014) e no ano seguinte, finalmente, é assinado o Acordo de Paris na COP 21. Dessa forma, o tema volta a ganhar atenção política na arena internacional. Internamente, ocorre a aprovação do Plano Nacional de Adaptação, em 2016, elaborado no âmbito do Grupo Executivo do Comitê Interministerial sobre Mudança do Clima, contando ainda com a contribuição de redes temáticas compostas por especialistas de vários setores.

Nesse período, as cidades brasileiras que aprovaram suas políticas climáticas foram: Recife-PE (2014), Sorocaba-SP (2016), Fortaleza-CE (2017) e Extrema- 
MG (2018). As cidades que publicaram seus planos de adaptação nessa fase foram: Porto Alegre-RS e Santos-SP (2016); Fortaleza-CE e Recife-PE (2019); Curitiba-PR, São Paulo-SP, Salvador-BA e Rio de Janeiro-RJ (2020). Como se pode notar, não são todas as cidades brasileiras com políticas climáticas que possuem um plano ou estratégia específica voltada para a adaptação.

Uma lacuna importante em relação às políticas climáticas locais no Brasil diz respeito às cidades costeiras. Elas são consideradas ainda mais vulneráveis às alterações climáticas por duas razões principais: pela sua especificidade geográfica, ou seja, sua interface entre continente, atmosfera e oceano e por se tratar de lugares com grande concentração de pessoas e estruturas, o que muda esses eventos para o status de desastres, uma vez que pessoas e estruturas podem ser afetadas de modo gravíssimo (IPCC, 2007; KRON, 2008). O Brasil possui uma costa de quase $7.500 \mathrm{~km}$ de extensão, onde muitas e algumas das mais importantes cidades do país se localizam e onde a maior parte da população está concentrada. E poucas cidades costeiras possuem uma estratégia de adaptação ou política climática: Fortaleza, Recife, Rio de Janeiro, Salvador e Santos.

Além das áreas costeiras, as regiões serranas também são consideradas vulneráveis aos efeitos das mudanças climáticas em virtude dos riscos de desmoronamento, deslizamentos e escorregamentos. Nesse sentido, em decorrência da tragédia da região serrana do Rio de Janeiro ${ }^{8}$, foi aprovada a Política Nacional de Proteção e Defesa Civil (Lei no 12.608), em 2012, que instituiu o Sistema Nacional de Proteção e Defesa Civil (SINPDEC). Por conseguinte, o enfoque das políticas públicas em âmbito federal passou da resposta ao pósdesastre e reconstrução para ações preventivas, que buscam principalmente salvaguardar a vida humana e vêm influenciando as políticas estaduais e municipais.

${ }^{8} \mathrm{O}$ desastre ocorreu entre os dias 11 e 12 de Janeiro de 2011, atingindo sete cidades da região serrana do Estado do Rio de Janeiro, quando fortes chuvas causaram enchentes e deslizamentos de terra, deixando mais de 900 mortos, cerca de 350 desaparecidos e milhares de desabrigados, além de graves danos à infraestrutura, economia e geografia da região. 
Assim, os planos de adaptação das cidades brasileiras têm envolvido principalmente os setores de defesa civil e planejamento urbano. No caso do Rio de Janeiro, a defesa civil municipal é responsável por boa parte das atividades relacionadas à adaptação, sendo diretamente ligada à gestão de desastres. Em São Paulo, as estratégias de adaptação envolvem a requalificação de moradias em áreas de risco e a recuperação de áreas de preservação permanente, a fim de prevenir ou minimizar os riscos de eventos climáticos extremos.

O plano de adaptação da cidade de Santos envolve doze eixos temáticos, com atenção especial para a zona costeira. A transversalidade também está presente no plano de Porto Alegre, que traz objetivos e iniciativas a fim de promover o aumento da resiliência da cidade, baseado no Enquadramento da Cidade Resiliente (City Resilience Framework) ${ }^{9}$.

A incorporação de projeções climáticas futuras nos planos de adaptação das cidades é fundamental para planejar o espaço urbano para as próximas décadas, a fim de garantir o melhor uso e ocupação desses espaços, a segurança das pessoas, bem como o desenvolvimento econômico e social. Nesse sentido, o plano de Santos considera as projeções climáticas para a região, resultantes das modelagens do projeto "Metropole"10 (NUNES et al., 2019).

As cidades brasileiras que possuem políticas climáticas encontram-se na transição entre o segundo e o terceiro estágio de adaptação às mudanças climáticas, conforme nos mostra a Figura 1.

\footnotetext{
${ }^{9}$ Esse enquadramento fornece uma lente para entender a complexidade das cidades e os fatores que contribuem para sua resiliência. Observar esses fatores pode ajudar as cidades a avaliar a extensão de sua resiliência, a identificar áreas críticas de fraqueza e a identificar ações e programas para melhorar a resiliência da cidade $<$ https://www.rockefellerfoundation.org/report/cityresilience-framework/ $>$, [última consulta em 10 de fevereiro de 2021].

10 O projeto Metropole "Uma estrutura integrada para analisar tomada de decisão local e capacidade adaptativa para mudança ambiental de grande escala: estudos de caso de comunidades no Brasil, Reino Unido e Estados Unidos" foi desenvolvido entre 2013 e 2017 por uma equipe científica multidisciplinar, nacional e internacional, composta por pesquisadores das seguintes instituições brasileiras: Centro Nacional de Monitoramento e Alertas de Desastres Naturais (Cemaden); Instituto Nacional de Pesquisas Espaciais (INPE); Universidade de São Paulo (USP); da Universidade Estadual de Campinas (Unicamp), Instituto Geológico de São Paulo (IG) e da Prefeitura de Santos.
} 
Figura 1 - Estágios de adaptação às mudanças climáticas nas cidades

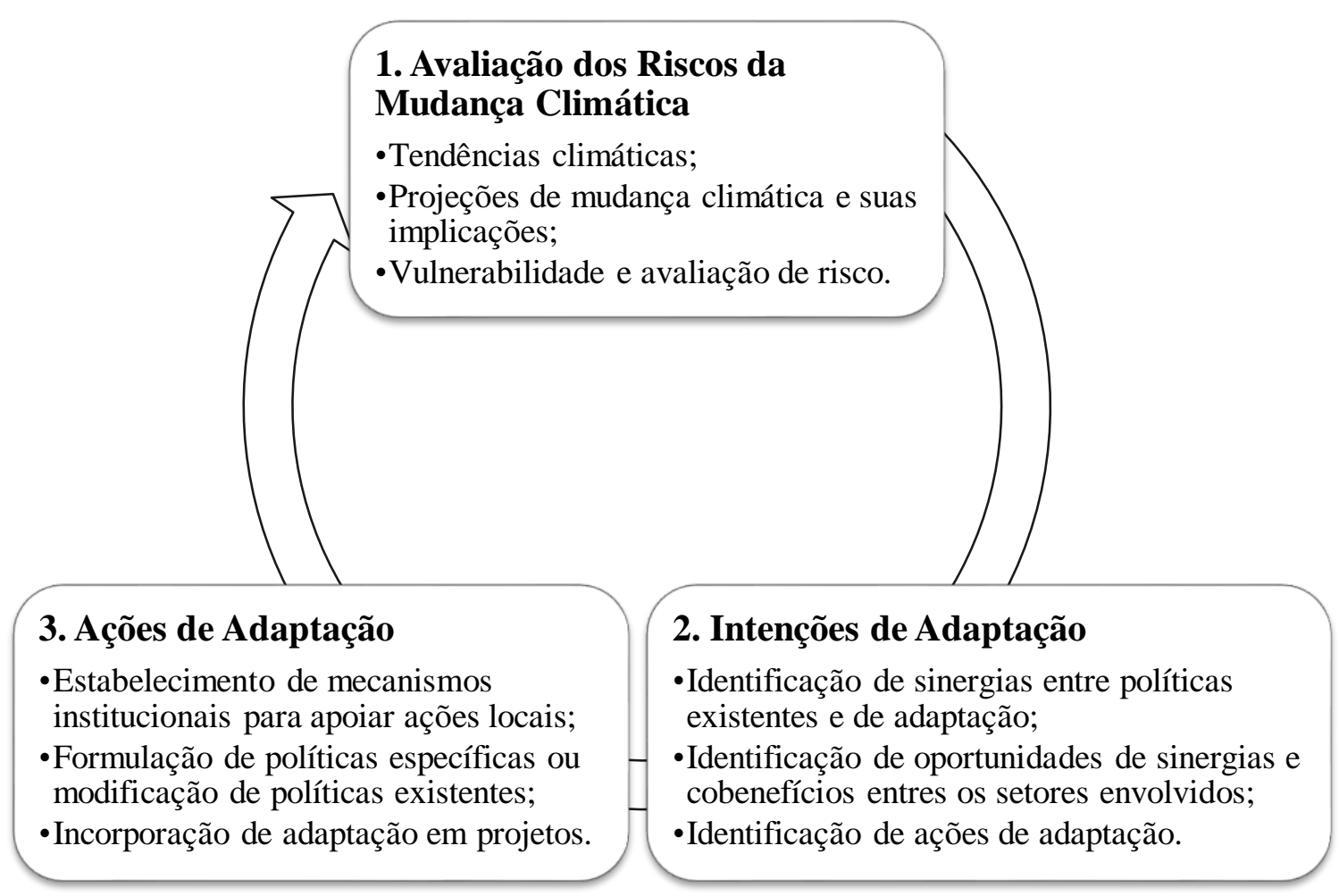

Fonte: BARBI (2015), baseado em GAGNON-LEBRUN, AGRAWALA (2006) e BASC (2010).

De acordo com a figura, o planejamento para adaptação começa com a avaliação das condições climáticas atuais e históricas, projeções de mudanças climáticas e as implicações futuras sobre as vulnerabilidades e impactos. Essas informações constituem a base das políticas de adaptação que podem ser formuladas como intenções de ação (2) ou ações de adaptação (3). As intenções incluem a identificação de possíveis ações de adaptação e de como elas podem se ajustar com outras políticas existentes. É nesse estágio que as cidades brasileiras com planos de adaptação se situam.

O passo seguinte, isto é, as ações de adaptação propriamente ditas referem-se ao estabelecimento de mecanismos institucionais para guiar e implementar as ações locais; a elaboração de políticas específicas ou ajustes nas políticas existentes e a incorporação explícita de medidas de adaptação no nível local. Ainda, essas 
ações de adaptação podem influenciar a avaliação de impactos futuros da mudança climática, a partir do monitoramento e avaliação dessas ações.

Um caminho possível e pouco explorado pelas cidades brasileiras se dá por meio da incorporação de estratégias de adaptação climática e redução das vulnerabilidades existentes e futuras da população e do território aos possíveis impactos da mudança climática no principal instrumento de planejamento urbano brasileiro: o plano diretor. Regulamentado pela Constituição da República de 1988 nos artigos 182 e 183 e pelo Estatuto da Cidade (Lei Federal 10.257/2001), o plano diretor orienta o desenvolvimento físico-territorial das cidades.

Nesse sentido, Espíndola e Ribeiro (2020) avaliam que os planos diretores de Belo Horizonte (BH), Campo Grande (MS), Salvador (BA), Rio Branco (AC) e Vitória (ES) apresentam contribuições indiretas, sem menção específica, em seus princípios e diretrizes, sobre possíveis políticas de adaptação ou à ampliação aos efeitos da mudança climática e destacam o plano diretor de Palmas (TO), que traz levantamentos e diretrizes específicos para as questões climáticas. Considerando que a sustentabilidade urbana ambiental, tal como a preservação e proteção do meio ambiente, são metas e diretrizes estabelecidas pelo próprio Estatuto da Cidade, a adaptação aos impactos das mudanças climáticas nas cidades deve passar pelos planos diretores.

\section{Agentes e arranjos institucionais para adaptação}

Além das RTMs, outro tipo de arranjo institucional que tem impulsionado a política climática nas cidades brasileiras são os comitês, fóruns e comissões municipais de mudanças climáticas, estabelecidas à semelhança do Fórum Brasileiro de Mudanças Climáticas (FBMC), criado em 2000, no âmbito federal.

O FBMC foi a primeira mobilização nacional em relação à questão climática que permitiu a articulação entre atores provenientes de diversos segmentos da sociedade. Com o objetivo de auxiliar o governo federal na incorporação das questões sobre mudanças climáticas nas políticas públicas, contou com a articulação de atores relevantes na área, provenientes de diversos ministérios e 
órgãos governamentais, representantes dos estados e instituições do setor privado e organizações da sociedade civil. Além de propiciar o debate acerca do tema e fornecer as bases para a Política Nacional de Mudança do Clima, um dos efeitos positivos do fórum foi a criação de quatorze fóruns estaduais de mudanças climáticas, permitindo o envolvimento de outros níveis de governo nessa questão. Esses fóruns foram os principais responsáveis pela aprovação das políticas climáticas nesses estados.

Esse tipo de arranjo institucional foi incorporado também no nível municipal de governo e teve um papel central na elaboração e aprovação das políticas climáticas municipais. A maior parte das cidades que possuem políticas climáticas aprovadas contam com fóruns ou comitês de mudanças climáticas, instituídos com o propósito de auxiliar o município a elaborar uma política específica para tratar dessa questão (ver Quadro 1).

Esses arranjos permitem a articulação entre diversas secretarias municipais e estaduais, iniciativa privada, instituições de ensino e pesquisa e o terceiro setor. Em todas as cidades que possuem plano de adaptação, esses fóruns ou comitês ficam a cargo da secretaria municipal de meio ambiente.

Quadro 1 - Arranjos institucionais relacionados às mudanças climáticas nas cidades brasileiras

\begin{tabular}{|c|c|c|c|}
\hline Município & Arranjo institucional & Secretaria relacionada & Ano de criação \\
\hline $\begin{array}{c}\text { Belo } \\
\text { Horizonte } \\
\text { (MG) }\end{array}$ & $\begin{array}{c}\text { Comitê Municipal sobre } \\
\text { Mudanças Climáticas e } \\
\text { Ecoeficiência }\end{array}$ & Meio Ambiente & $\begin{array}{c}2006 \\
\text { (Decreto no } \\
12.362)\end{array}$ \\
\hline $\begin{array}{c}\text { Curitiba } \\
\text { (PR) }\end{array}$ & $\begin{array}{c}\text { Fórum Curitiba sobre } \\
\text { Mudanças Climáticas }\end{array}$ & Meio Ambiente & $\begin{array}{c}\text { (Atualizado pelo } \\
\text { Decreto } \mathrm{n}^{\circ} \\
\text { 498/2016) }\end{array}$ \\
\hline
\end{tabular}




\begin{tabular}{|c|c|c|c|}
\hline $\begin{array}{l}\text { Fortaleza } \\
\qquad(\mathrm{CE})\end{array}$ & $\begin{array}{l}\text { Fórum de Mudanças } \\
\text { Climáticas }\end{array}$ & $\begin{array}{l}\text { Urbanismo e Meio } \\
\text { Ambiente }\end{array}$ & $\begin{array}{c}2015 \\
\text { (Decreto } n^{\circ} \\
13.639)\end{array}$ \\
\hline $\begin{array}{l}\text { Porto } \\
\text { Alegre } \\
\text { (RS) }\end{array}$ & $\begin{array}{l}\text { Comitê Municipal de } \\
\text { Mudanças Climáticas e } \\
\text { Eficiência Energética }\end{array}$ & Meio Ambiente & $\begin{array}{c}2016 \\
\left(\text { Decreto } n^{\circ}\right. \\
19.348)\end{array}$ \\
\hline Recife (PE) & $\begin{array}{c}\text { Comitê de Sustentabilidade } \\
\text { e Mudanças Climáticas }\end{array}$ & $\begin{array}{l}\text { Meio Ambiente e } \\
\text { Sustentabilidade }\end{array}$ & 2013 \\
\hline $\begin{array}{c}\text { Rio de } \\
\text { Janeiro (RJ) }\end{array}$ & $\begin{array}{c}\text { Fórum Carioca sobre } \\
\text { Mudança do Clima e } \\
\text { Desenvolvimento } \\
\text { Sustentável }\end{array}$ & Meio Ambiente & $\begin{array}{c}2009 \\
\text { (Decreto } n^{\circ} \\
31415)\end{array}$ \\
\hline Santos (SP) & $\begin{array}{l}\text { Comissão Municipal de } \\
\text { Adaptação à Mudança do } \\
\text { Clima }\end{array}$ & $\begin{array}{c}\text { Desenvolvimento Urbano } \\
\text { (em 2019, passou para o } \\
\text { Meio Ambiente) }\end{array}$ & $\begin{array}{c}2015 \\
\left(\text { Decreto } n^{\circ}\right. \\
7.293)\end{array}$ \\
\hline $\begin{array}{l}\text { São Paulo } \\
\text { (SP) }\end{array}$ & $\begin{array}{l}\text { Comitê Municipal de } \\
\text { Mudança do Clima e } \\
\text { Ecoeconomia }\end{array}$ & Meio Ambiente & $\begin{array}{c}2005 \\
\text { (Decreto } n^{\circ} \\
45.959)\end{array}$ \\
\hline $\begin{array}{l}\text { Sorocaba } \\
\text { (SP) }\end{array}$ & $\begin{array}{c}\text { Comitê Gestor Local sobre } \\
\text { Mudanças Climáticas }\end{array}$ & $\begin{array}{c}\text { Meio Ambiente, Parques e } \\
\text { Jardins }\end{array}$ & $\begin{array}{c}2019 \\
\left(\text { Decreto } n^{\circ}\right. \\
24.515)\end{array}$ \\
\hline
\end{tabular}

Fonte: Elaborado pelos autores.

Esses fóruns e comitês se reúnem periodicamente e grande parte deles criaram grupos de trabalho, com atribuições e temas específicos ligados às mudanças climáticas. O tema de adaptação, na maior parte das cidades, fica a cargo desses grupos de trabalho. Além disso, esses arranjos são importantes para assegurar a implementação das políticas aprovadas, em teoria. Em Salvador, um arranjo formado por agentes não governamentais - ambientalistas, lideranças comunitárias e cientistas, representando coletivos, ONGs, territórios, laboratórios e 
institutos de pesquisa, foi criado em 2020 com o propósito de acompanhar a implementação do plano climático na cidade.

A maioria dos planos trata a questão da adaptação de forma multissetorial na sua implementação, característica necessária ao enfrentamento do problema, que não é exclusivamente de responsabilidade do setor ambiental, permeando diferentes setores governamentais (UN-HABITAT, 2011). Este ponto demonstra uma maior compreensão acerca da questão climática, que no caso da adaptação envolve também os setores de desenvolvimento urbano, meio ambiente construído, infraestrutura e serviços urbanos, sequestro de carbono, gestão de desastres e saúde.

A dinâmica interna das estruturas governamentais no nível local também é importante para a governança climática. Em muitas cidades, a questão climática ainda está concentrada no departamento de meio ambiente (KERN, ALBER, 2008; UN-HABITAT, 2011). Isso limita a capacidade do governo local por duas razões: primeiro, os departamentos de meio ambiente são muitas vezes marginalizados dentro das estruturas governamentais do município e podem conflitar com outras partes da administração local; segundo, a natureza transversal da questão climática não permite que os departamentos de meio ambiente sejam capazes de implementarem sozinhos as políticas necessárias para tratar o tema (BULKELEY, 2010). Nesse contexto, a integração, coordenação e cooperação através das agências e departamentos governamentais são fundamentais. Em algumas cidades, isso tem funcionado com a criação de uma estrutura administrativa e institucional específica para coordenar as políticas climáticas.

Outro ponto de destaque em relação a esses arranjos institucionais é a possibilidade de estreitamento do diálogo entre cientistas e pesquisadores e os gestores públicos e tomadores de decisão. As estratégias de adaptação dependem muito do conhecimento científico acerca das mudanças climáticas, pois é necessário saber ao que, de fato, adaptar-se. Nesse sentido, os esforços de projeções climáticas, riscos e mudanças na escala local, de maneira mais detalhada, são fundamentais para apoiar as políticas e ações de adaptação. 
A produção científica acerca das mudanças climáticas no Brasil aumentou significativamente nas últimas décadas. As consequências das mudanças climáticas estão bem documentadas e há melhores informações e uma melhor compreensão dos riscos e impactos futuros. Ainda assim, esse conhecimento encontra-se muitas vezes fragmentado, para as escalas locais, apesar dos esforços importantes de sistematização desses estudos para escalas regionais, como é o caso dos relatórios do Painel Brasileiro de Mudanças Climáticas (PBMC, 2013).

A necessidade de conhecer melhor os riscos locais para planejar suas ações de adaptação fez com que a Comissão Municipal de Adaptação à Mudança do Clima de Santos criasse uma Comissão Consultiva Acadêmica (CCA). A CCA conta com pesquisadores e cientistas, especialmente com estudos e pesquisas na região e tem o objetivo de agregar suas contribuições para a atual revisão do plano de adaptação do município. Nessa perspectiva, a cidade de Santos tem se posicionado mais próxima do estágio 3 (ver Figura 1), em que políticas específicas de adaptação estão sendo formuladas, bem como a incorporação de ações de adaptação em políticas existentes, como o Plano Diretor, por exemplo.

\section{CONSIDERAÇÕES FINAIS}

As mudanças climáticas se configuram como uma condição a ser enfrentada pelas cidades em todo o mundo. A agenda internacional de adaptação aos impactos das mudanças climáticas tem sido reforçada pelos Objetivos do Desenvolvimento Sustentável e pelo Acordo de Paris. Nesse trabalho, analisou-se como essa agenda chega às cidades brasileiras, isto é, como elas estão respondendo aos riscos das mudanças climáticas em termos de ações governamentais de adaptação.

As políticas locais de adaptação são fundamentais na busca pela redução da vulnerabilidade socioambiental em cada parte do país, uma vez que os impactos das mudanças climáticas são diferentes para cada localidade. Isso é de particular importância diante do atual governo federal brasileiro, que adota uma posição 
negacionista em relação às mudanças climáticas. Nessas circunstâncias, os níveis estadual e municipal de governo podem assumir o papel de liderar as ações climáticas no país, bem como garantir que as contribuições nacionais pretendidas sejam atendidas de acordo com o Acordo de Paris.

Os resultados apresentados mostraram que a agenda de adaptação é recente nas cidades brasileiras. Todos os planos de adaptação foram publicados a partir de 2016. No caso brasileiro, as RTMs trouxeram a questão climática para agenda política municipal e impulsionaram a capacidade de governança das mudanças climáticas dos governos locais brasileiros. Para as cidades de Porto Alegre, Rio de Janeiro, Salvador e São Paulo, que integram mais que uma dessas redes, elas tiveram um papel central na agenda local de adaptação.

Os resultados também sinalizaram que os comitês, fóruns e comissões municipais de mudanças climáticas tiveram um papel significativo na elaboração e aprovação das políticas climáticas, que culminaram no avanço da agenda de adaptação na maior parte das cidades brasileiras.

Conforme foi exposto, as estratégias de adaptação dependem muito do conhecimento científico acerca das mudanças climáticas e no nível local envolvem diversos setores de atividades, como obras públicas, planejamento, meio ambiente, saúde pública, serviços e gestão de desastres. Essas iniciativas não deveriam se resumir a redesenhar construções e infraestruturas. Para tanto, é necessário o conhecimento local, uma participação maior de outros agentes provenientes de outros segmentos da sociedade e maior capacidade institucional dos governos locais, pontos que ainda são desafios para grande parte das cidades brasileiras.

Concomitantemente, é preciso relacionar as respostas às mudanças climáticas às questões de desenvolvimento e promover o desenvolvimento das cidades considerando as estratégias de mitigação e adaptação. Nesse caminho, um dos maiores desafios que as cidades brasileiras têm pela frente é uma compreensão mais eficiente do enfrentamento dos riscos climáticos, o que requer manter a perspectiva entre escalas e reconhecer a natureza multinível e multidimensional 
das mudanças climáticas. Esse não é um desafio unicamente ambiental, tratado isoladamente de outras questões econômicas e sociais. As mudanças climáticas nas áreas urbanas interferem em uma série extensa de desafios políticos existentes e emergentes, entre eles estão a irradicação da pobreza, saneamento, escassez de água e alimentos e crescimento populacional. Quando a mudança climática for abrangida como parte integrante desses desafios, as soluções poderão ser mais adequadas para refletir e tratar a miríade de impactos nas cidades.

\section{REFERÊNCIAS}

ADGER, W.N.; HUQ, S.; BROWN, K.; CONWAY, D.; HULME. M. "Adaptation to climate change in the developing world", in Progress in Development Studies, v.3, n.1, p. 179-195, 2003.

ADGER, W.N., DESSAI, S., GOULDEN, M. et al. "Are there social limits to adaptation to climate change?", in Climatic Change, v. 93, pp. 335-354, 2009.

AGRAWAL, A. The Role of Local Institutions in Adaptation to Climate Change. The World Bank. 2008. https://doi.org/10.1596/28274.

AMBRIZZI, T.; LACERDA, C. B. "A Ciência do Clima e a Sustentabilidade", in RIBEIRO, W. C. (Org.) Governança da Ordem Ambiental Internacional e Inclusão Social. São Paulo: AnnaBlume Editora e Comunicação, p. 237-257, 2012.

BARBI, F. Mudanças climáticas e respostas políticas nas cidades. Campinas, Brazil: Editora da Unicamp, 2015.

BARBI, F.; MACEDO, L. V. "Transnational municipal networks and cities in climate governance: Experiments in Brazil”, in VAN DER HEIJDEN, J., BULKELEY, H., CERTOMÀ, C. (eds.) Urban Climate Politics: Agency and Empowerment. Cambridge University Press. The Earth System Governance Series. 2019.

BASC - BOARD ON ATMOSPHERIC SCIENCES AND CLIMATE. Adapting to the Impacts of Climate Change. America's Climate Choices: Panel on Adapting to the Impacts of Climate Change. Washington: The National Academies Press. 2010. 
BELLINSON, R. G. "Connecting the dots: The politics of governing urban climate adaptation innovations through transnational municipal networks", in HUGHES, S.; CHU, E. K., MASON, S. G. (Eds.) Climate Change in Cities: Innovations in MultiLevel Governance. Springer International Publishing. The Urban Book Series. 2018.

BETSILL, M., BULKELEY, H. "Transnational networks and global environmental governance: The cities for climate protection program", in International Studies Quarterly, v. 48, n.2, pp. 471-493, 2004.

BOUTELIGIER, S. "Multinational Companies and Urban Climate Governance: Market making or Successful Policy Innovation?", in CRAIG, J., TOLY, N., SCHROEDER, $\mathrm{H}$. (eds.) The Urban Climate Challenge - Rethinking the Role of Cities in the Global Climate Regime. London: Routledge. 2015.

BRASIL. Decreto de 28 de agosto de 2000. Dispõe sobre o Fórum Brasileiro de Mudanças Climáticas e dá outras providências. 2000.

BRASIL. Pretendida Contribuição Nacionalmente Determinada para Consecução do Objetivo da Convenção-Quadro das Nações Unidas sobre Mudança do Clima. Disponível em http://www.itamaraty.gov.br/images/ed_desenvsust/BRASIL-iNDCportugues.pdf, acesso em 08/01/2020. 2015.

BULKELEY, H. "Cities and the Governing of Climate Change", in Annual Review of Environment and Resources, v.35, n.1, pp. 229-253, 2010.

BULKELEY, H.; BETSILL, M. Cities and Climate Change: Urban Sustainability and Global Environmental Governance. New York: Routledge, 2003.

BULKELEY, H.; BETSILL, M. M. "Revisiting the urban politics of climate change", in Environmental Politics, v.22, n.1, pp. 136-154, 2013.

BULKELEY, H., EDWARDS, G., FULLER, S. "Contesting climate justice in the city: Examining politics and practice in urban climate change experiments", in Global Environmental Change, v.25, n.1, pp. 31-40, 2014.

BULKELEY, H.; NEWELL, P. Governing Climate Change. New York: Routledge, 2010. 
CONTIPELLI, E. "Multi-level Climate Governance: Polycentricity and Local Innovation", in Revista Catalana de Dret Ambiental, Vol. 9, Núm. 2, 2018.

DI GIULIO, G. M., LAPOLA, D., TORRES, R. et al. "Plano Nacional de Adaptação à Mudança do Clima: possibilidades e desafios", in Jornal da Ciência. Disponível em:

<http://www.jornaldaciencia.org.br/edicoes/?url=http://jcnoticias.jornaldaciencia.org .br/24-plano-nacional-de-adaptacao-a-mudanca-do-clima-possibilidades-edesafios/>, última consulta em 10 de fevereiro de 2021. 2016.

ESPÍNDOLA, I. B.; RIBEIRO, W. C. "Cidades e mudanças climáticas: desafios para os planos diretores municipais brasileiros", in Cad. Metrop., São Paulo, v. 22, n. 48, pp. 365-395, maio/ago 2020.

FBMC - FÓRUM BRASILEIRO DE MUDANÇAS CLIMÁTICAS. Balanço das Atividades - 2004-2010. 2011.

FERREIRA, L. C. "A busca de alternativas de sustentabilidade no poder local", in FERREIRA, L. C. e VIOLA, E. (orgs.) Incertezas de sustentabilidade na globalização. Campinas: Universidade Estadual de Campinas, 1996.

FÜNFGELD, H. "Facilitating local climate change adaptation through transnational municipal networks", in Current Opinion in Environmental Sustainability, v.12, n.1, pp. 67-73, 2015.

GAGNON-LEBRUN, F., AGRAWALA, S. Progress on Adaptation to Climate Change in Developed Countries. An Analysis of Broad Trends. OECD, 2006.

GIDDENS, A. The Politics of Climate Change. Cambridge: Polity Press, 2009.

GORDON, D.; ACUTO, M. "If cities are the solution, what are the problems? The promise and perils of urban climate leadership", in JOHNSON, C.; TOLY, N.; SCHROEDER, H. (eds.) The urban climate challenge: Rethinking the role of cities in the global climate regime. Routledge: New York. 2015.

HALL, P.; PFEIFFER, U. Urban Future 21: A Global Agenda for Twenty-First Century Cities. New York: E\&FN Spon, 2013. 
HAPPAERTS, S., VAN DEN BRANDE, K., BRUYNINCKX, H. Governance for sustainable development at the inter-subnational level: The case of the Network of Regional Governments for Sustainable Development (nrg4SD), Working paper n`3, February 2008.

HARVEY, D. "Tackling Urban $\mathrm{CO}_{2}$ Emissions in Toronto", in Environment, v.35, n.7, pp.16-20; 38-44. 1993.

HEGGER, D. L. T., MEES, H. L. P., DRIESSEN, P. P. J., RUNHAAR, H. A. C. "The roles of residents in climate adaptation: A systematic review in the case of the Netherlands", in Environmental Policy and Governance, v. 27, n. 4, pp. 336-350, 2017.

HEIDRICH, O., RECKIEN, D., OLAZABAL, M. et al. "National climate policies across Europe and their impacts on cities strategies", in J. Environ. Manag., n.168, pp. 36-45, 2016.

HEYNEN, N. "The urban century", in Progress in Human Geography, v. 38, n. 4, 598-604, 2014.

HICKMANN, T., FUHR, H; HÖHNE, C.; LEDERER, M.; STEHLE, F. "Carbon Governance Arrangements and the Nation-State: The Reconfiguration of Public Authority in Developing Countries", in Public Administration and Development, v.37, n.5, pp. 331-343, 2017.

HUQ, S.; KOVATS, S.; REID, H.; SATTERTHWAITE, D. "Editorial: Reducing risks to cities from disasters and climate change", in Environment and Urbanization, v. 19, n.3, pp. 3-15, 2007.

IBGE - Instituto Brasileiro de Geografia e Estatística. Censo 2010. 2010.

IEA - Global Energy Review 2021, IEA, Paris. Disponível em: https://www.iea.org/reports/global-energy-review-2021, última consulta em 20 de abril de 2021. 2021.

IPCC - INTERGOVERNMENTAL PANEL ON CLIMATE CHANGE. Summary for Policymakers. In: Climate Change 2007: Mitigation. Contribution of Working Group III to the Fourth Assessment Report of the Intergovernmental Panel on Climate 
Change [B. Metz, O.R. Davidson, P.R. Bosch, R. Dave, L.A. Meyer (eds.)], Cambridge University Press. 2007.

IPCC - INTERGOVERNMENTAL PANEL ON CLIMATE CHANGE. Summary for policymakers. In: Climate Change 2014: Impacts, Adaptation, and Vulnerability. Part A: Global and Sectoral Aspects. Contribution of Working Group II to the Fifth Assessment Report of the Intergovernmental Panel on Climate Change [Field, C.B., V.R. Barros, D.J. Dokken, K.J. Mach, M.D. Mastrandrea, T.E. Bilir, M. Chatterjee, K.L. Ebi, Y.O. Estrada, R.C. Genova, B. Girma, E.S. Kissel, A.N. Levy, S. MacCracken, P.R. Mastrandrea, and L.L.White (eds.)]. Cambridge University Press, Cambridge, United Kingdom and New York, NY, USA, pp. 1-32. 2014.

KERN, K., ALBER, G. "Governing Climate change in cities: Modes of urban Climate governance in multi-level systems", in Competitive Cities and Climate Change, OECD Conference Proceedings, Milan, Italy, pp. 171-192. 2008.

KLEIN, R. J.T., ADAMS, K. M., DZEBO, A., et al. Advancing climate adaptation practices and solutions: Emerging research priorities. Stockholm Environment Institute, Working Paper n. 2017-07, 2017.

KRON, W. Coasts: The riskiest places on Earth. In: Coastal Engineering. Proceedings of the 31st International Conference. V.1. Hamburg, Germany, 2008.

LINDSETH, G. "Local level adaptation to climate change: discursive strategies in the Norwegian context", in Journal of Environmental Policy and Planning, v. 7, n. 1, pp. 61-83, 2005.

MAGRIN, G.O., MARENGO, J.A., BOULANGER, J.-P. et al. Central and South America. In: Climate Change 2014: Impacts, Adaptation, and Vulnerability. Part B: Regional Aspects. Contribution of Working Group II to the Fifth Assessment Report of the Intergovernmental Panel on Climate Change [Barros, V.R., C.B. Field, D.J. Dokken, M.D. Mastrandrea, K.J. Mach, T.E. Bilir, M. Chatterjee, K.L. Ebi, Y.O. Estrada, R.C. Genova, B. Girma, E.S. Kissel, A.N. Levy, S. MacCracken, P.R. Mastrandrea, and L.L.White (eds.)]. Cambridge University Press, pp. 1499-1566. 2014. 
MEASHAM, T., PRESTON, B., SMITH, T., et al. "Adapting to climate change through local municipal planning: barriers and challenges", in Mitigation and Adaptation Strategies for Global Change, v.16, n. 8, pp 889-909, 2011.

MEES, H. "Local governments in the driving seat? A comparative analysis of public and private responsibilities for adaptation to climate change in European and North-American cities", in Journal of Environmental Policy and Planning, v. 19, n. 4, pp. 374-390, 2017.

NUNES, L.H., GRECO, R.; MARENGO, J.A. (eds.) Climate Change in Santos, Brazil: Projections, Impacts and Adaptation Options. Springer Nature Switzerland, 2019.

OLSSON, L., OPONDO, M., TSCHAKERT, P., et al. Livelihoods and poverty. In: Climate Change 2014: Impacts, Adaptation, and Vulnerability. Part A: Global and Sectoral Aspects. Contribution of Working Group II to the Fifth Assessment Report of the Intergovernmental Panel on Climate Change [Field, C. B., V. R. Barros, D. J. Dokken, K. J. Mach, M. D. Mastrandrea, T. E. Bilir, M. Chatterjee, K. L. Ebi, Y. O. Estrada, R. C. Genova, B. Girma, E. S. Kissel, A. N. Levy, S. MacCracken, P. R. Mastrandrea, and L. L. White (eds.)]. Cambridge University Press, 793-832, 2015. PBMC - Painel Brasileiro de Mudanças Climáticas. Base Científica das Mudanças Climáticas. 1ํ. Relatório de Avaliação Nacional. Volume 1, 2013.

RASHIDI, K.; PATT, A. "Subsistence over symbolism: the role of transnational municipal networks on cities' climate policy innovation and adoption", in Mitig Adapt Strateg Glob Change, v.23, n.1, pp. 507-523, 2018.

REI, F.C.F., CUNHA, K.B., SETZER, J. "La Paradiplomacia Ambiental en la Nueva Governanza Internacional", in Revista TIP, Buenos Aires, n.2, p. 50-63, 2012.

REI, F. C. F. "International Environmental Law and Global Environmental Governance: Southern Influences", in Veredas do Direito: Direito Ambiental e Desenvolvimento Sustentável, Belo Horizonte, v. 15, n. 32, p. 143-165, 2018. 
RIBEIRO, W. C. "Riscos e vulnerabilidade urbana no Brasil", in Scripta Nova. Revista Electrónica de Geografía y Ciencias Sociales. Barcelona: Universidad de Barcelona, vol. XIV, n. 331, 2010.

RYAN, D. "From commitment to action: a literature review on climate policy implementation at city level", in Climatic Change, v.131, n.1, pp. 519-529, 2015.

SATTERTHWAITE, D. Climate change and urbanization: Effects and implications for urban governance. Texto preparado para "United Nations Expert Group Meeting on Population Distribution, Urbanization, Internal Migration and Development", 21-23 de janeiro 2008, New York, UNDESA. 2008.

SCHROEDER, H.; BULKELEY, H. "Global Cities and the Governance of Climate Change: What is the Role of Law in Cities?", in Fordham Urban Law Journal, v.36, n.2, pp.314-360, 2009.

SETZER, J. "Governança multinível das mudanças climáticas: políticas subnacionais e ações transnacionais em São Paulo", in REI, F.C.F. (org.) Direito e desenvolvimento: Uma abordagem sustentável. São Paulo: Saraiva, 2013.

TOLY, N. J. "Transnational municipal networks in climate politics: from global governance to global politics", in Globalizations, v. 5, n. 3, pp. 341-356, 2008.

UNFCCC - United Nations Framework Convention on Climate Change. 2015. Adoption of the Paris Agreement. Disponivel em: https://unfccc.int/resource/docs/2015/cop21/eng//09r01.pdf, acesso em 05/08/2019.

UN-HABITAT (United Nations Human Settlements Programme). Cities and climate change: global report on human settlements. Earthscan. 2011.

UN-HABITAT. World Cities Report 2016. Nairobi: UN-Habitat. 2016.

VAN DER HEIJDEN, J. "City and Subnational Governance", in JORDAN, A., HUITEMA, D., VAN ASSELT, H. et al., Governing Climate Change: Polycentricity in Action?, Cambridge University Press, 2018. 
VICTOR, D. G. "Making the promise of Paris a reality", in STAVINS, R. N.; STOWE, R. C. (eds). The Paris Agreement and Beyond: International Climate Change Policy Post-2020. Cambridge, Mass: Harvard Project on Climate Agreements, 2016. 